\title{
Water flow meter measurement uncertainties
}

\author{
R. P. Dias ${ }^{1}$, J. G. Dalfré Filho ${ }^{1}$ \& Y. F. L. de Lucca ${ }^{2}$ \\ ${ }^{1}$ College of Civil Engineering, Architecture and Urbanism - UNICAMP, \\ Brazil \\ ${ }^{2}$ Hydraulics Technological Center - CTH-DAEE-USP and UNICAMP, \\ Brazil
}

\begin{abstract}
In water supply systems, managers need to get water from a source and supply an entire population. Knowledge regarding the flow of water is a critical parameter within these systems, thus the need for flow meters. When flow meters are placed near pipeline singularities, the velocity profile changes and can cause measurement errors. Due to these potential errors, measurement norms and standards are created to require flow meters to be placed in rectilinear-shaped pipelines for a more accurate measurement. However, managers claim that in field measurement situations, it is not always possible to meet these standards, and consequently, adjusting the error from the acquired data is necessary. Therefore, this study investigates how the error in the flow rate varies with the distance from singularities when using an ultrasonic flow meter, which, as a nonintrusive measurement method, is easily installed on the outside walls of the pipeline. This flow meter was installed at different distances from an elbow and the measurements obtained were compared to those from an electromagnetic flow meter, which accumulate accurate data when installed at standard conditions. Comparing the data from those two flow meters, it was possible to obtain the variation of error as a function of flow and distance from the singularity.
\end{abstract}

Keywords: uncertainties, ultrasonic flow meter, water supply systems.

\section{Introduction}

The water flow is an essential technical parameter that needs to be known by the management staff of every water supply plant. This information allows the staff to capture the water from the source, treat it and supply it to the population. In an 
environment where water is scarce and demand is high, a precise control of water flow is extremely important. In order for this control to be achieved, flow meters must be employed. However, those flow meters usually work in continuous periods, and from time to time they need to be calibrated. That calibration becomes difficult when they are installed inside pipelines because they need to be removed and transported to a certified lab for a precise calibration. This represents an inconvenience and, moreover, requires the system to be completely shut down to accommodate the maintenance of the device. With that being said, the employment of meters that are external to pipelines is both an interesting and potentially valuable option.

For a good precision in data acquired by the flow meter, a straight length upstream from where it is installed is needed to avoid changes in the velocity profile caused by singularities like curves and valves. ISO-9104 [1] requires a straight length in the order of 10 diameters upstream of the flow meter, however in many cases, it is not possible to meet this criteria. One example is the case where water reuse systems are attached to pre-existing installations.

Because water flow control is related to waste and spills, it directly impacts the budget of companies and the balance of the natural environment. Seeking to avoid negative economic and environmental impacts, this experimental research addresses water flow measurements in a hydraulic system, employing an ultrasonic flow meter, which presents effective performance and can be installed externally to pipelines. The ultrasonic meter was installed at different positions from one singularity. An electromagnetic meter was employed to calibrate the ultrasonic meter and to determine measurement error. The electromagnetic device was installed according to all criteria required by technical standard measurements. The goal of this research was to determine the uncertainties derived from water flow measurement when an ultrasonic meter was employed in straight lengths that are smaller than those required by normal standards.

\section{Literature review}

Flow meters are to be selected based on the following: type of fluid to be measured, loss of charge, precision, available room for installation, temperature, operational pressure, reliability and cost. All the flow meters have uncertainties in measurements, but, prior to determining those uncertainties, it is necessary to describe the types of errors that can occur.

According to ISO-5168 [2] and Vuolo [3], three types of errors exist: random errors, which can be due to a large number of variables and cannot be controlled by the researcher, and that cause measurements to differ from the true value in a constant manner; systematic errors, which occur when all measures differ from the true value in a constant manner; and spurious errors, caused by human error or problems within the device. Systematic errors can be minimized with instrument calibration. Additionally, even when environmental factors do not interfere with measurement, it is customary to take note of the highest possible number of these factors, such as temperature, pressure, and humidity. 
The goal of the measurement is to determine the specific measure of the target variable. According to Kamal [4], in general, this process will only be completed when the uncertainties are determined. The number of significant digits must also be calculated and should be used to represent both the experimental standard deviation and the mean standard deviation. According to ISO-5168 [2] and Miller [5], if the systematic error is equal to zero, the flow Q given by eqn. (1) will include the true mean approximately $95 \%$ of the time:

$$
Q=\bar{Q} \pm t_{95} \cdot s_{\bar{Q}}
$$

where: $\bar{Q}=$ Mean flow; $\mathrm{t}_{95}=$ Student $\mathrm{T}$ value, $\alpha=95 \%$, dependent on degrees of freedom $(\mathrm{df}=\mathrm{n}-1) ; s_{\bar{Q}}=$ mean standard deviation of the flow.

Additionally, according to Miller [5], the systematic error $\mathrm{B}_{\mathrm{i}}$ can be calculated through the relationship between the mean and arbitrary value as the reference value. In the present study, the systematic error was calculated through the comparison between the mean flow from the ultrasonic meter and the mean flow from the electromagnetic meter (reference value), according to eqn. (2):

$$
B_{i}(\%)=\frac{Q_{i, u}-Q_{i, e}}{Q_{i, e}} .100
$$

where: $\mathrm{B}_{\mathrm{i}}=$ systematic error of situation $\mathrm{i}$ (in percentage); $\mathrm{Q}_{\mathrm{i}, \mathrm{u}}=$ mean flow in the ultrasonic meter in situation $\mathrm{i}$; $\mathrm{Q}_{\mathrm{i}, \mathrm{e}}=$ mean flow in the electromagnetic meter in situation i.

Commonly, the correction factor $F_{B}$ is used to adjust readings when a systematic error is calculated using eqn. (2). The mean value can be corrected when it is multiplied by the value of $F_{B}$ given by eqn. (3):

$$
F_{B}=\left(1+\frac{B}{100}\right)^{-1}
$$

Uncertainty, $\mathrm{U}$, in a measurement can be calculated by combining the random and systematic errors in order to determine a confidence interval. It's considered a standard deviation in the systematic error and a standard deviation in the random error. Therefore, eqn. (4) is obtained.

$$
U=B \pm \sqrt{\left(t_{95} s_{x i}\right)^{2}+\frac{\left(t_{95} s_{x i}\right)^{2}}{(\sqrt{n})^{2}}}
$$

where: $\mathrm{U}=$ uncertainty; $\mathrm{B}=$ systematic error; $\mathrm{t}_{95}=$ Student $\mathrm{T}$ value; $\mathrm{n}=$ sample size; $\mathrm{s}_{\mathrm{xi}}=$ experimental standard deviation.

If the value of the systematic error is known, each measurement needs to be corrected with the correction factor $\mathrm{F}_{\mathrm{B}}$, in which case the uncertainty, $\mathrm{U}$, is given by eqn. (5) below:

$$
U= \pm \sqrt{\left(1+\frac{1}{n}\right)\left(t_{95} s_{x i}\right)^{2}}
$$


In this experiment, the variations of the systematic and random errors were determined in the case when an ultrasonic meter was installed near singularities.

\section{Experimental facility}

This research was performed at the Laboratory of Hydraulics and Fluid Mechanics at the College of Civil Engineering, Architecture and Urbanism at UNICAMP, located in Campinas, Brazil. The test bench was constructed using two straight pipeline pieces which interconnect through two 90-degree curves. The flow meters were installed within the straight pieces. Two valves were employed to control the flow, as demonstrated in fig. 1.

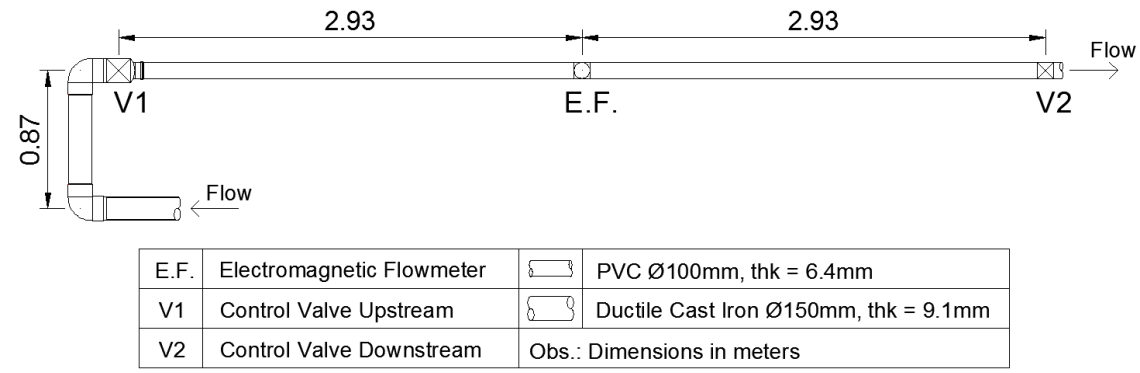

Figure 1: $\quad$ Testing Bench diagram.

The electromagnetic flow meter employed in the study is a 4” Model 570TM by Fisher-Rosemount from Brazil, with uncertainty equal to $1 \%$ of the measured value. This meter was installed in the straight length section as demonstrated in fig. 1, in accordance with the norms, criteria and suggestions by ISO-9104 [1], Delmée [6], Spink [7], Troskolanski [8] and Souza and Bortoni [9]. The ultrasonic flow meter is a TransPortTM Model PT868 Portable Flowmeter by Panametrics. According to the manufacturer's manual, its uncertainty value depends on the pipeline diameter and on the velocity of the fluid, whose values are presented in table 1 :

Table 1: $\quad$ Typical error values for the Ultrasonic Flowmeter model PT868 by Panametrics.

\begin{tabular}{|c|c|c|}
\hline & Speed $<0.3 \mathrm{~m} / \mathrm{s}$ & Speed $>0.3 \mathrm{~m} / \mathrm{s}$ \\
\hline Diameter $>150 \mathrm{~mm}(6 ”)$ & $\pm 0.01 \mathrm{~m} / \mathrm{s}$ & $2 \%$ (1\% if calibrated) \\
\hline Diameter $\leq 150 \mathrm{~mm}(6 ”)$ & $\pm 0.05 \mathrm{~m} / \mathrm{s}$ & $2 \%$ to $5 \%$ \\
\hline
\end{tabular}

The ultrasonic meter was installed at 10 different positions from valve V1 upstream, as shown in fig. 1. For each position of the flow meter, tests were conducted for 20 different flows, at a flow interval between 0 and 1,000 1/min. For each test, a reading of the flows was performed in the ultrasonic and 
magnetic meters, and the results were recorded for future analysis. The measuring distances from the valve were as follows: $1 D_{\text {int }}=98 \mathrm{~mm}, 2 \mathrm{D}_{\text {int }}=$ $196 \mathrm{~mm}, 3 \mathrm{D}_{\text {int }}=294 \mathrm{~mm}, 4 \mathrm{D}_{\text {int }}=392 \mathrm{~mm}, 5 \mathrm{D}_{\text {int }}=490 \mathrm{~mm}, 6 \mathrm{D}_{\text {int }}=588 \mathrm{~mm}, 7 \mathrm{D}_{\text {int }}=$ $686 \mathrm{~mm}, 8 \mathrm{D}_{\text {int }}=784 \mathrm{~mm}, 9 \mathrm{D}_{\text {int }}=882 \mathrm{~mm}$ and $10 \mathrm{D}_{\text {int }}=980 \mathrm{~mm}$.

Per the manufacturer's recommendation, the ultrasonic meter was installed with the two transducers placed on the same horizontal line, at the upper side of the pipeline. Water temperature was considered to be at $20^{\circ} \mathrm{C}$. Testing began once all relevant parameters for the test were established.

\section{Results and analysis}

When repeated flow readings are performed in a lab, the obtained values are subject to random errors and remain at a certain average for a permanent flow regimen. Maximum and minimum values were obtained for each flow range established during 30 seconds for each position of the ultrasonic meter. The mean flow $\mathrm{Q}_{\mathrm{i}}$ was then calculated, creating the arithmetic mean of the maximum and minimum flows at the established flow range.

For the distance of $1 \mathrm{D}_{\text {int, }}$ it was observed that, even for distances near to valve V1 upstream, the ultrasonic meter generated reasonable results as when compared to the flow results generated by the electromagnetic meter used as a reference. However, for this specific distance and flow of 1,000 1/min, two equipment errors occurred, E1 (Low Signal) and E2 (Sound Speed Error), and as a result no readings were recorded. When the flow was decreased through valve V2 downstream to approximately $9501 / \mathrm{min}$, the equipment no longer presented reading errors, and the readings were performed. It is probable that the equipment presented this error because four traverses were used, which caused the transducers to be located further apart. It is important to remember that a traverse represents each crossing made by the ultrasonic wave, from one side to the other side of the pipeline. If the transducers are installed on the same side of the pipeline, there will always be an even number of traverse. Another factor to be considered is that the fluid speed in the $1,0001 / \mathrm{min}$ flow was higher, which caused more turbulence and interfered with flow measurement.

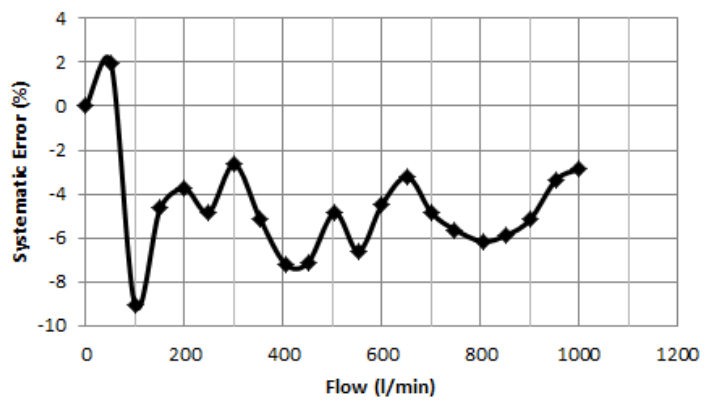

Figure 2: $\quad$ Systematic flow error variation at a distance of $10 \mathrm{D}_{\text {int }}$. 
Having the mean flow of each meter, and establishing that the mean obtained from the electromagnetic meter is the flows reference value, it is possible to determine the systematic error present in each measurement by using eqn. 2 . On calculating the systematic error for each distance and different flows, graphs were prepared to represent how the systematic error varies with the flow. The graph in fig. 2 presents this variation at a distance of $10 \mathrm{D}_{\text {int }}$ from the valve.

The tendency for the systematic error to increase or decrease as the flow increases was not present. The same was found true as the distance decreases, the tendency for the systematic error to increase or decrease was not present. Fig. 3 presents the maximum, minimum and mean values between distances for each flow. Fig. 4 presents the maximum, minimum and mean values between flows for each distance.



Figure 3: Maximum, minimum and mean values of the systematic error between distances according to flow.



Figure 4: Maximum, minimum and mean values of the systematic error between the 20 measured flows according to distance.

In fig. 3, a flow of 50 1/min generated the highest systematic error which also corresponds to the distance of $1 \mathrm{D}_{\text {int }}$. This plot is out of the range of mean values, 
and this situation could have been caused either by a measurement error or as a consequence of the low speed along with the short distance from the valve. It should also be noted that for low flow values, a small difference in values causes a relatively large systematic error.

Because the systematic error is independent from both the distance to the valve and from the flow, it is possible to obtain a mean, a mean standard deviation, the number of significant digits and the calibration factor that can be employed in the equipment, according to ISO-5168 [2]. These referred values are described in table 2. There were a total of 400 flow points which were obtained in the lab. Therefore, one can apply the Student T-value of 2.0, according to Miller [5], in order to calculate a standard deviation with 95\% confidence.

Table 2: $\quad$ Final values of the systematic error for the Ultrasonic meter.

\begin{tabular}{|l|l|}
\hline Mean Systematic Error & $\mathrm{B}=-3.236 \%$ \\
\hline Systematic Error Standard Deviation & $\mathrm{S}_{\mathrm{B}}= \pm 0.159 \%$ \\
\hline $\begin{array}{l}\text { Mean Systematic Error Standard Deviation } \\
\text { with the Application of Student T }\end{array}$ & $t_{95}{ }^{\mathrm{S}_{\mathrm{B}}}= \pm 0.319 \%$ \\
\hline Significant Digits & 0.003 \\
\hline Mean Systematic Error Final Value & $\mathrm{B}=-3.236 \pm 0.319 \%$ \\
\hline Calibration Factor & 1.03344 \\
\hline
\end{tabular}

One can notice that the Systematic Error Value is close to the Maximum Value of the uncertainty of the instrument, which is $5 \%$, as presented in table 1 . Hence, the calibration factor can be used in the ultrasonic meter in order to improve measurement precision.

Relating to random error, fig. 5 and fig. 6 were prepared and present a comparison between the standard deviation in relation to the mean values including flows and distances. Fig. 5 presents the maximum, minimum and mean values of the standard deviation between the distances for each flow point. Fig. 6 presents the maximum, minimum and mean values of the standard deviation between flows for each distance.

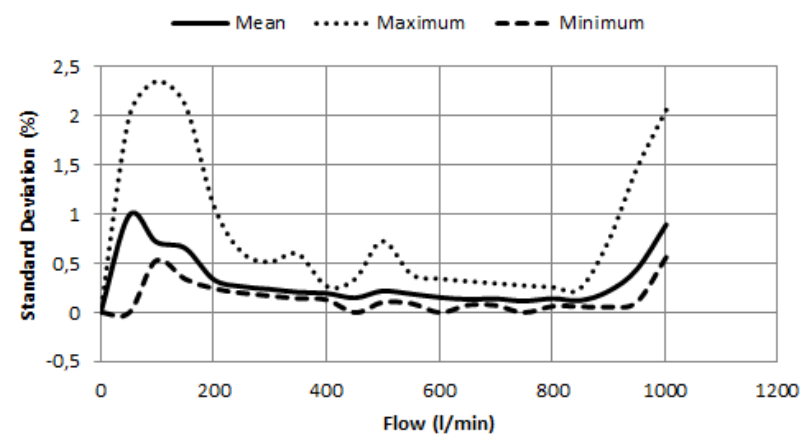

Figure 5: Maximum, minimum and mean values of the standard deviation between distances for each flow point. 


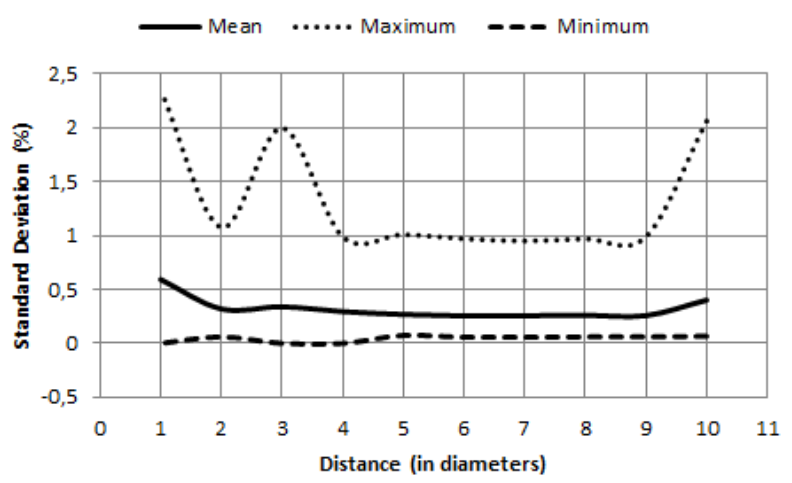

Figure 6: Maximum, minimum and mean values of the standard deviation between flows for each distance.

The graph in fig. 5 shows a higher standard deviation for the low flows. This can be explained due to the fact that even one digit that varies represents a significant portion of the total. A constant random error of approximately $0.17 \%$ was obtained between readings of $300-8501 / \mathrm{min}$, along with a tendency of increase in the random error as the flow surpasses the value of $8501 / \mathrm{min}$.

On the contrary, the graph in fig. 6 demonstrates that the random error is independent of the distance to a specific singularity, remaining at an average of $0.33 \%$. Some maximum points appear to be discrepant, which could have been caused by an installation problem, despite all the caution taken.

The worst-case scenario of the relative standard deviation occurred with a flow around 1,000 1/min, for which 27 values were recorded. Hence, it has a Tdistribution value of 2.056, in accordance with Miller [5]. The results are presented in table 3 .

Table 3: Random error final values of the ultrasonic meter for the worst-case scenario.

\begin{tabular}{|l|l|}
\hline $\begin{array}{l}\text { Relative Experimental Standard } \\
\text { Deviation }\end{array}$ & $\mathrm{s}_{\mathrm{xi}}= \pm 1.3 \%$ \\
\hline $\begin{array}{l}\text { Final Value of the Random Error after } \\
\text { Employment of the T-distribution }\end{array}$ & $t_{95}{ }^{*} \mathrm{~s}_{\mathrm{xi}}= \pm 2.6 \%$ \\
\hline Significant Digits & 0.2 \\
\hline
\end{tabular}

By knowing the equipment's random and systematic errors, it is possible to calculate the total uncertainty, U, through the use of eqn. (4). The results found in this case was an uncertainty, $U$, between $-5.855 \%$ and $-0.617 \%$ of the measurement value. In case every measurement is calibrated with the calibration factor, the uncertainty is then between $-2.619 \%$ and $2.619 \%$ of the flow value, for the critical case in which the flow equals $1,0001 / \mathrm{min}$. This value is within 
the uncertainty range of the equipment, described in the manufacturer's manual as being between $\pm 2 \%-5 \%$ of the flow value.

When analyzing the central range of the graph in fig. 5, with flow values between $300 \sim 850 \mathrm{l} / \mathrm{min}$, table 4 is obtained through the same method described above. In this case, 12 flow intervals with 3 points each for 10 different distances were adopted for a total of 360 points, with T-value equal to 2.0.

Table 4: $\quad$ Ultrasonic meter random error final values for flow range between $3001 / \mathrm{min}$ and $8501 / \mathrm{min}$.

\begin{tabular}{|l|l|}
\hline Relative Standard Deviation & $\mathrm{s}_{\mathrm{xi}}= \pm 1.01 \%$ \\
\hline $\begin{array}{l}\text { Final Value of the Random Error after } \\
\text { Applying the T-Distribution }\end{array}$ & $t_{95}{ }^{*} \mathrm{~s}_{\mathrm{xi}}= \pm 2.02 \%$ \\
\hline Significant Digits & 0.04 \\
\hline
\end{tabular}

The results for uncertainty, $\mathrm{U}$, in this case show an uncertainty between $-5.281 \%$ and $-1.191 \%$ of the measurement value. In each case where the measurement is calibrated with the calibration factor, the uncertainty lies between $-2.045 \%$ and $2.045 \%$ of the flow value for a flow range between 300 $\mathrm{l} / \mathrm{min}$ and $850 \mathrm{l} / \mathrm{min}$. By calibrating the meter, the uncertainty obtained is exactly at the lower range of the uncertainty of the equipment, described in the manufacturer's manual. This value corresponds to $\pm 2 \%$ of the flow value.

Therefore, the ultrasonic meter does present a systematic error, but when the obtained calibration factor is employed, measurement values become more precise. It was also verified that the systematic error does not vary according to the flow, nor does it vary according to the distance to the singularity, which in this case is a valve. On the other hand, the random error does vary according to the flow, although it does not vary according to the distance to the valve. There is a range in which the random error is shown to be constant, and when a flow value of 850 1/min was achieved, this error tended to increase. When applying a calibration factor to the measurements, it is safe to say that the uncertainty value will be $\pm 2.619 \%$ in the most critical scenario where the flow is approximately $1,0001 / \mathrm{min}$. At the same time, for a flow range between $3501 / \mathrm{min}$ and 850 $1 / \mathrm{min}$, the total uncertainty drops to a value of $\pm 2.045 \%$.

\section{Conclusion}

It can be concluded that flow meters present both systematic and random errors that affect the total uncertainty of the collected data. In the case of the ultrasonic meter, it was demonstrated that the systematic error does not vary according to the distance to a valve, nor does it vary with the flow. It was also demonstrated that the meter's random errors are constant at a specific flow range, and increase as the flow increases, as the random error showed to be dependent on fluid velocity. However, the random error is not dependent on the distance variable.

It was also noted that the devices need to be adequately calibrated before they are installed, if they are to be used in real-world, non-controlled environments. 
With this calibration, the uncertainty range of measurements can be decreased, which is made possible by calculating the systematic error in comparison to a standard meter used as a reference. In the case of facilities where the length of straight stretches does not meet the 10 diameters upstream and five diameters downstream that are required by the standards for ultrasonic meters, it is still possible to achieve adequate precision, given the conditions described by this article.

However, it is not possible to affirm that a measurement yielded by an ultrasonic meter will present systematic errors when installed near different singularities. In order to investigate that, future studies need to be developed.

\section{References}

[1] International Organization For Standardization - ISO 9104. Measurement of fluid flow closed conduit methods of evaluating the performance of electromagnetic flow meter for liquids. Geneva, Switzerland, 18 p., 1991.

[2] International Organization For Standardization - ISO 5168. Measurement of fluid flow: Procedures for the evaluation of uncertainties. Geneva, Switzerland, 65 p., 2005.

[3] Vuolo, J. H. Fundaments of error theory. São Paulo: E. Blucher, 225 p., 1992.

[4] Kamal, I.A.R. Measurement and Instrumentation Techniques. Ed. do autor, 369p., 2000.

[5] Miller, W.R., Flow measurement engineering handbook. 3rd edition. New York: McGraw-Hill, 1168 p., 1996.

[6] Delmée, G. J., Manual of flow measurement. 2nd edition. São Paulo: Ed. Edgard Blücher, 476 p., 1983.

[7] Spink, L.K. Principles and practice flow meter engineering. Foxborough, Mass: Foxboro Company, 575 p., 1967.

[8] Troskolanski, A.T., Hydrometry: theory and practice of hydraulic measurement. New York: Pergamon Press, 684 p., 1960.

[9] Souza, Z.; Bortoni, E.C., Instrumentation for Energetic and Industrial Systems. 1st edition. Itajubá: Ed. Novo Mundo, 387 p, 2006. 